\title{
Cirugía para el manejo del síndrome de apnea e hipopnea obstructiva del sueño y de la roncopatía: Revisión de 71 casos clínicos
}

\author{
Surgery in the management of obstructive sleep apnea and \\ hypopnea syndrome and snoring: Review of 71 clinical cases
}

Alejandro 0jeda $S^{1}$, Javiera Pardo J², Michelle Arroyo $D^{3}$, Daniel Muñoz $S^{4}$, Carlos Stott $C^{1}$.

\begin{abstract}
RESUMEN
Introducción: El síndrome de apnea e hipoapnea obstructiva del sueño (SAHOS) es una patología altamente prevalente y se asocia a importante comorbilidad cardiovascular. Su etiología es multifactorial. Dentro de las opciones terapéuticas, la cirugía de la vía aérea superior es una alternativa efectiva en casos seleccionados.

Objetivo: Describir la experiencia del Servicio de Otorrinolaringología del Hospital Clínico de la Universidad de Chile en cirugía de pacientes con diagnóstico de SAHOS.

Material y método: Estudio retrospectivo descriptivo. Se analizan fichas clínicas, antecedentes demográficos y mórbidos, polisomnografía (PSG), tipo de cirugía, puntaje de Epworth pre y posoperatorio, estadía hospitalaria, complicaciones y seguimiento.

Resultados: De un total de 71 pacientes (87\% de sexo masculino, edad promedio de 44 años), el 88\% tiene diagnóstico preoperatorio de sobrepeso u obesidad. Al $67 \%$ de los pacientes se les realizó PSG, en el 64\% de ellas se demostró un SAHOS moderado a severo. En el $97 \%$ de los casos se realizó uvulopalatoplastía con radiofrecuencia (UPP), en el 62\% septoplastía (SP) y en el 50\% amigdalectomía. En el 98\% de los pacientes se asociaron 2 a 4 técnicas, destacando la SP más UPP como la combinación más frecuente (15,45\%). El promedio de estadía hospitalaria fue de 1,09 días. Sólo el 7\% de los pacientes presentó complicaciones. El seguimiento promedio fue de 3,05 meses.

Discusión: El rol de la cirugía es eliminar el colapso de la vía aérea superior, lo que se logra en la mayoría de los pacientes usando técnicas asociadas.

Conclusión: La indicación de cirugía en SAHOS debiera combinar diferentes técnicas dependiendo de la localización anatómica de la obstrucción y el tipo de paciente a intervenir.
\end{abstract}

Palabras clave: Cirugía, apnea del sueño.

\footnotetext{
${ }^{1}$ Médico. Servicio de Otorrinolaringología, Hospital Clínico Universidad de Chile.

${ }^{2}$ Médico. Servicio de Otorrinolaringología, Hospital Barros Luco Trudeau.

${ }^{3}$ Médico. Servicio de Otorrinolaringología, Hospital San Juan de Dios.

${ }^{4}$ Médico. Universidad de Chile.
} 


\section{ABSTRACT}

Introduction: The obstructive sleep apnea syndrome (OSA) is a highly prevalent disease and is associated with significant cardiovascular morbidity. Its etiology is multifactorial. Among the therapeutic options, surgery of the upper airway is an effective alternative in selected cases.

Aim: To describe the experience of the Otorhinolaryngology Service of Hospital of the University of Chile in surgery of patients with OSA.

Material and method: Retrospective descriptive study. We analyzed clinical and morbid records, demographic background, polysomnography (PSG), type of surgery, pre and post surgical Epworth scores, hospital stay, complications and follow-up.

Results: Of a total of 71 patients ( $87 \%$ male, average age of 44 years), $88 \%$ had a preoperative diagnosis of overweight or obesity. $67 \%$ of patients were done a PSG, $64 \%$ of them showed a moderate to severe OSA. 97\% of patients underwent radiofrequency uvulopalatoplasty (UPP), 62\% septoplasty (SP) and 50\% tonsillectomy. In 98\% of patients, 2 to 4 associated techniques were used, highlighting the association of UPP plus $S P$ as the most frequent combination (15.45\%). The average hospital stay was 1.09 days. Only $7 \%$ of patients had complications. The average follow-up time was 3.05 months.

Discussion: The role of surgery is to remove the collapse of the upper airway, which is achieved in most patients using associated techniques.

Conclusion: Surgery for OSA should include different techniques combination, anatomical location of obstruction and patient's profile.

Key words: Surgery, sleep apnea.

\section{INTRODUCCIÓN}

El síndrome de Apnea e Hipopnea Obstructiva del Sueño (SAHOS) se define como una alteración anatómica y funcional de la vía aérea superior (VAS) que genera episodios repetidos de obstrucción completa (apnea) o parcial (hipopnea) de ésta, por el colapso de las partes blandas de la faringe durante el sueño ${ }^{1}$. Estos episodios de obstrucción de la VAS se miden a través de una polisomnografía (PSG), con el índice de apneashipopneas (IAH). Un IAH $>5$ asociado a síntomas relacionados con la enfermedad y no explicados por otras causas, confirma el diagnóstico².

Por otro lado, a diferencia del SAHOS, la roncopatía es una entidad clínica caracterizada por la presencia de ruidos producidos por la vibración del paladar blando y de los pilares posteriores durante el sueño. Su diagnóstico no precisa de la realización de PSG y reviste mayor importancia cuando se asocia a SAHOS ${ }^{3}$.

En el SAHOS, la obstrucción episódica de la VAS provoca descensos de la saturación de oxígeno arterial, despertares transitorios ${ }^{1}$ y un sueño no reparador, lo que se manifiesta con somnolencia diurna excesiva y trastornos cognitivo-conductuales, respiratorios, cardíacos, inflamatorios y metabólicos diversos. Este síndrome se asocia además con una elevada morbimortalidad cardiovascular (especialmente con un IAH >15), alteración de la calidad de vida y accidentes automovilísticos ${ }^{2,4}$.

EI SAHOS afecta aproximadamente al $20 \%$ de los adultos, con distintos grados de severidad. Los factores de riesgo de esta patología incluyen edad avanzada ( $4^{\mathrm{a}}-7^{\mathrm{a}}$ década), obesidad, género masculino, consumo de alcohol y tabaco, uso de sedantes y vía aérea superior anatómicamente pequeña ${ }^{5}$.

Los tratamientos actuales para el SAHOS son diversos e incluyen: fármacos, uso de dispositivos intraorales para adelantar la posición mandibular, uso de presión positiva continua de aire en la vía aérea superior (CPAP) para mantenerla neumáticamente abierta y cirugía ${ }^{6}$. Esta última, consiste principalmente en procedimientos quirúrgicos a nivel de los tejidos blandos orofaríngeos (tonsilectomía, adenoidectomía, uvulopalatofaringoplastía, avance geniogloso con miotomía y suspensión del hioides, linguoplastía, glosectomía media, 
epiglotoplastía y exéresis de lesiones patológicas obstructivas) ${ }^{7}$.

La cirugía en SAHOS busca aliviar la sintomatología, mejorar la calidad de vida y reducir los signos polisomnográficos de severidad ${ }^{8}$.

Nuestro objetivo, fue describir la experiencia del Servicio de Otorrinolaringología del Hospital Clínico de la Universidad de Chile (HCUCH) en cirugía de pacientes con diagnóstico de SAHOS y/o roncopatía.

\section{MATERIAL Y MÉTODO}

Se realizó un estudio retrospectivo, descriptivo y analítico de todos los pacientes con indicación de cirugía por síndrome de apnea obstructiva del sueño, operados entre los años 2008 a 2010 en el Servicio de Otorrinolaringología del HCUCH. Se analizan los antecedentes demográficos y mórbidos, polisomnografía (PSG), tipo de cirugía, puntaje de Epworth pre y posoperatorio, estadía hospitalaria, complicaciones y seguimiento. La información fue extraída de fichas clínicas y del archivo de la Unidad de Enfermedades del Sueño $\mathrm{HCUCH}$.

A modo analítico, se condujo un modelo de regresión lineal múltiple para verificar la relación entre el número de eventos hora en la polisomnografía y la influencia del sexo, edad y estado nutricional, a modo de identificar él o los factores de riesgo más importantes. Se utilizó la prueba exacta de Fisher para comparar variables categóricas y el test $t$ de Student para muestras pareadas al comparar variables continuas. Para todo test de hipótesis se consideró como significativo un valor $\mathrm{p}$ menor a 0,05 . Los datos fueron analizados mediante el software estadístico Stata 10.1 (StataCorp. 2008. College Station, TX: StataCorp LP).

\section{RESULTADOS}

Se analizó un total de 71 fichas clínicas, obteniéndose que $87,3 \%(\mathrm{n}=62)$ de los pacientes fue de sexo masculino y $12,7 \%(n=9)$ de sexo femenino. La edad promedio fue de 44,5 años (rango entre 20 y 63 años) para la muestra general, siendo de $50,3 \pm 6,8$ años para las mujeres y de $43,7 \pm 10,4$ años para los hombres ( $p=0,035$ ).

El estado nutricional previo del grupo de pacientes en estudio de acuerdo al IMC fue de: sobrepeso $54,9 \%$, obesidad $33,8 \%$ y normal el $11,26 \%$.

Al $67 \%$ de los pacientes se les realizó evaluación polisomnográfica preoperatoria y en $64 \%$ de ellos se demostró SAHOS moderado a severo, con un promedio 36,3 eventos de apnea por hora de sueño (índice de apnea e hipopnea) en la PSG.

El análisis sobre la influencia de los factores de riesgo arrojó que solamente el peso se relacionó significativamente con el índice de apnea e hipopnea $(p=0,001)$, mientras que las variables edad $(p=0,585)$, sexo $(p=0,708)$, presencia de hipertensión arterial $(p=0,124)$, diabetes mellitus ( $p=0,807)$ y dislipidemia $(p=0,51)$, no presentaron relación significativa con este puntaje.

Las técnicas quirúrgicas empleadas fueron: en el $97,2 \%$ de los casos uvulopalatoplastía con radiofrecuencia (UPP), en el $62 \%$ septoplastía (SP) y en el $49,3 \%$ amigdalectomía (Figura 1).

En el $98 \%$ de los pacientes se utilizaron 2 a 4 técnicas asociadas (Figura 2), destacando la SP más UPP como la combinación más frecuente, representando el $15,45 \%$ de los casos (Figura 3 ).

El promedio de estadía hospitalaria fue de 1,09 días. Sólo el 7\% de los pacientes presentó complicaciones siendo el sangrado posoperatorio el más frecuente. El seguimiento promedio fue de 3,05 meses.

El puntaje Epworth preoperatorio promedio fue de 16,5 puntos (moderada somnolencia diurna) y el posoperatorio de 3 puntos (sin somnolencia diurna), siendo esta diferencia estadísticamente significativa $(p=0,002)$.

\section{DISCUSIÓN}

EI SAHOS es una condición patológica que se asocia a un importante incremento de la morbimortalidad. Se ha relacionado con aumento de la prevalencia de hipertensión arterial, enfermedades cardiovasculares, somnolencia diurna y deterioro de la calidad de vida. Muchos pacientes responden a modificaciones de hábitos de vida, como reducción de peso, cambios posicionales al 


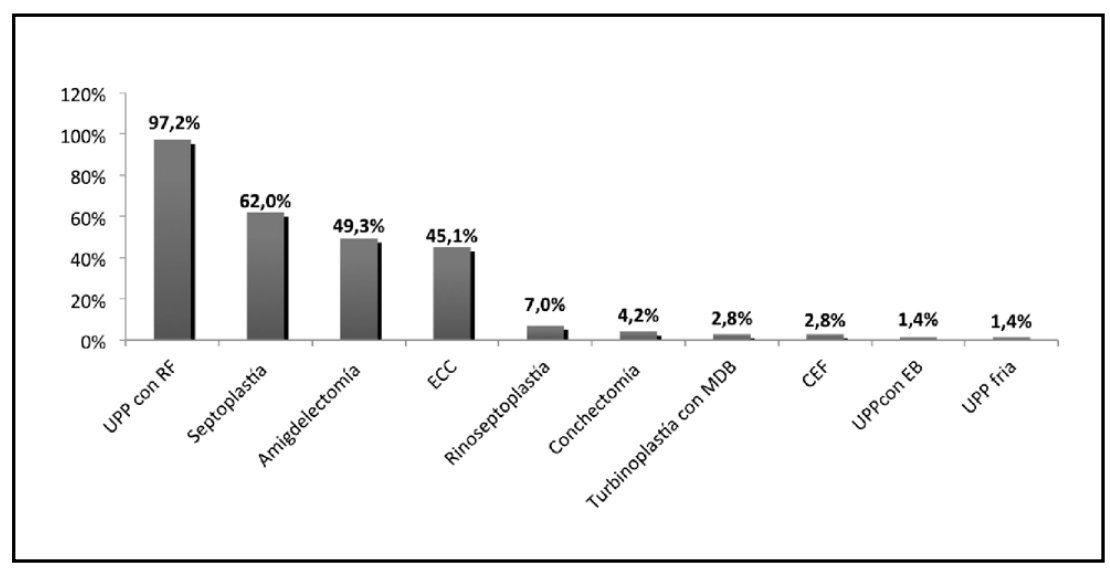

Figura 1. Distribución según porcentaje de técnicas quirúrgicas empleadas.

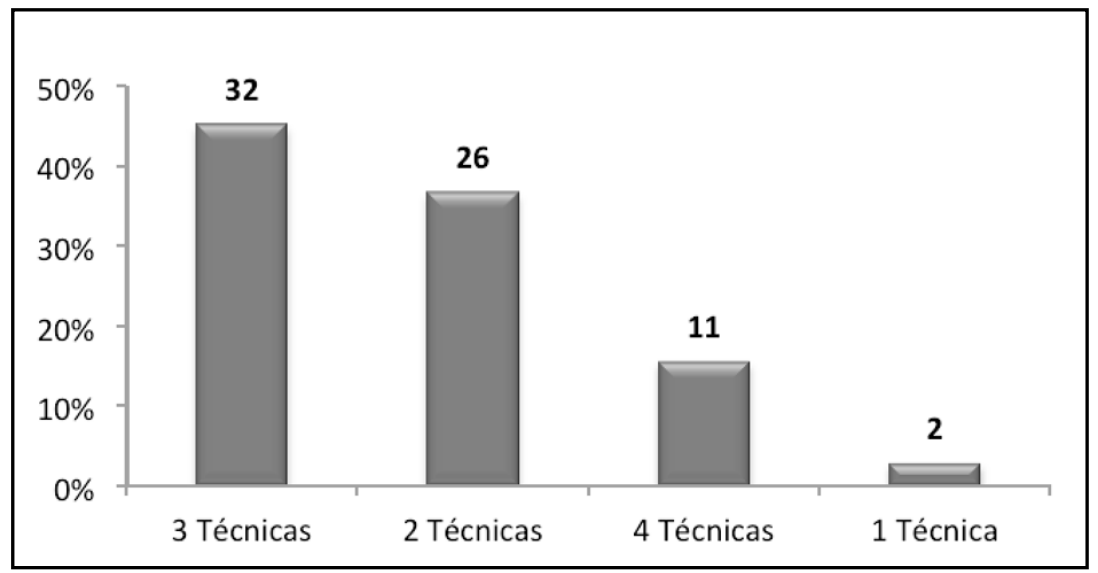

Figura 2. Distribución según número de técnicas quirúrgicas asociadas empleadas.

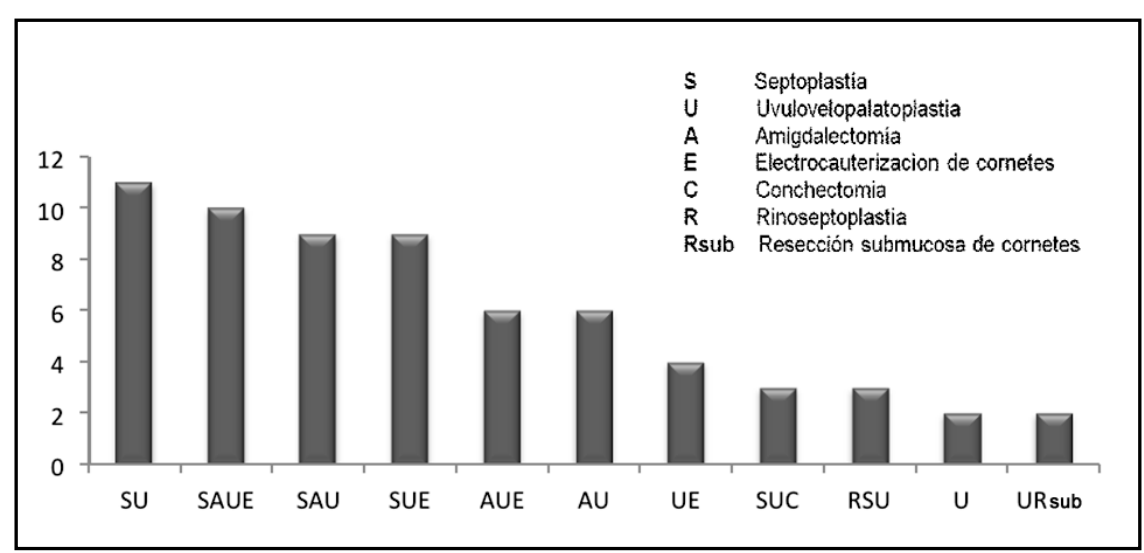

Figura 3. Distribución según número de combinaciones de técnicas quirúrgicas empleadas. 
dormir, reducción de la ingesta de alcohol y sedantes, prótesis orales e implementación de CPAP. Aunque el CPAP es actualmente considerado como el tratamiento de elección, este método cuenta con escasa aceptación a largo plazo dentro de este grupo de pacientes. El manejo quirúrgico entonces aparece como una real alternativa cuando la terapia médica fracasa 0 presenta escasa tolerancia y adherencia. Su objetivo básicamente es lograr un lumen que mantenga una adecuada permeabilidad del tracto respiratorio superior durante el sueño. Las técnicas quirúrgicas destinadas a corregir las anormalidades anatómicas del tracto respiratorio superior, han demostrado ser exitosas en el alivio de la sintomatología diurna y sobre todo en la reducción de la intensidad del ronquido durante el sueño, pero muestran discretos resultados en la mejoría de los episodios obstructivos respiratorios nocturnos.

Al estudiar la experiencia del HCUCH en cirugía en pacientes con SAHOS y/o roncopatía, se observa un marcado predominio de pacientes de sexo masculino y con malnutrición por exceso (sobrepeso y obesidad), lo que concuerda con la literatura disponible.

La técnica más empleada es la UPP, sin embargo, destaca ampliamente la asociación de técnicas por sobre el empleo de técnicas aisladas, siendo la UPP más SP la combinación más prevalente.

En el presente estudio destaca una mejoría clínica estadísticamente significativa de los pacientes, apreciable en el cambio promedio del puntaje Epworth. Al contrario, estudios extranjeros sobre la efectividad de la UPP en SAHOS, revelan una persistencia importante de la patología después de la cirugía al evaluar el IAH pre y posquirúrgico $0^{9}$. Sin embargo, estos datos no son comparables, dado que empleamos un indicador distinto a los demás estudios, por no disponerse de evaluación polisomnográfica de los pacientes después de la intervención quirúrgica. El estudio de los resultados polisomnográficos posoperatorios es entonces un objetivo pendiente.

\section{CONCLUSIÓN}

EI SAHOS es una patología prevalente no exenta de complicaciones. Su manejo sigue siendo tema de discusión y análisis, por lo que revisar el empleo de técnicas quirúrgicas y su efectividad, es de real importancia para identificar los factores que impiden que la cirugía tenga un rol eficaz en su tratamiento.

El éxito de la cirugía para el manejo del SAHOS dependería directamente de la correcta elección del paciente, determinado principalmente por una obstrucción anatómica demostrable por examen físico y exámenes imagenológicos pertinentes, por la confirmación diagnóstica con PSG y determinación de la severidad del cuadro clínico, comorbilidad asociada y fracaso previo del manejo médico.

\section{BIBLIOGRAFÍA}

1. LeE KJ, ed. Essential Otolaryngology, Head and Neck Surgery. $9^{\text {th }}$ ed. Mc Graw Hill, 2008.

2. Lloberes P, Durán-Cantolla J, Martínez-García Ma, Marín JM, Ferrer a, Corral J, Masa JF, Parra 0, Alonso-Álvarez ML, Terán-Santos J. Diagnosis and treatment of sleep apneahypopnea síndrome. Arch Bronconeumol 2011; 47(3): 143-56.

3. Teculescu D, Hannhart B, Aubry C, MontautVerient B, Virion JM, Michaely JP, Gueguen R. Who Are the "Occasional" Snorers? Chest 2002; 122: 562-8.

4. Aurora Rn, Casey kR, Kristo D, Auerbach S, Bista SR, Chowdhuri S, Karippot A, Lamm C, Ramar K, Zak R, Morgenthaler TI; American Academy of Sleep Medicine. Practice parameters for the surgical modifications of the upper airway for obstructive sleep apnea in adults. Sleep 2010; 33(10): 1408-13.

5. Durán J, Esnaola S, Rubio R, iztueta A. Obstructive sleep apnea-hipopnea and related clinical features in a population-based sample of subjects eight aged 30 to $70 \mathrm{yr}$. Am J Respir Crit Care Med 2001; 163: 685-9.

6. Woodson BT. Non-pressure therapies for obstructive sleep apnea: surgery and oral appliances. Respir Care 2010; 55(10): 1314-21.

7. Sundaram S, Lim J, Lasserson TJ. Surgery for obstructive sleep apnoea in adults. Cochrane Database of Systematic Reviews. In: The 
Cochrane Library, Issue 4, Art. No. CD001004. DOI: 10.1002/14651858.CD001004.

8. Sunitha C, Kumar SA. Obstructive sleep apnea and its management. Indian J Dent Res 2010; 21(1): 119-24.
9. Caples S, Rowley J, Prinsell J, Pallanch J, Elamin M, Katz S, Harwick J. Surgical Modifications of the Upper Airway for Obstructive Sleep Apnea in Adults: A Systematic Review and Meta-Analysis. Sleep 2010; 33(10): 1396-407. 